\title{
Separation of Phenol from Bio-oil Produced from Pyrolysis of Agricultural Wastes
}

\author{
Zeban Shah ${ }^{1}$, Renato CV1, Marco AC ${ }^{1}$, Rosangela DS \\ ${ }^{1}$ Federal University of Rio, Grande do Sul, Av. Bento Gonçalves Porto Alegre, RS, Brazil \\ ${ }^{2}$ Pontifical Catholic University of Rio Grande do Sul, Av Ipiranga Porto Alegre, RS, Brazil
}

\begin{abstract}
The aim of this study was to separate phenol from Bio-Oil obtained from the pyrolysis of agricultural wastes (BAW). The BAW was obtained in one step catalytic pyrolysis in which temperature of the reactor was kept at $30^{\circ} \mathrm{C}$ and then increased up to $900^{\circ} \mathrm{C}$. After pyrolysis, the BAW was distillated and analyzed by Gas chromatography and Mass spectrometry (GC-MS) technique and comprehensive two-dimensional gas chromatography with time-of-flight mass spectrometry detection (GC $\times$ GC/TOFMS) Where BAW showed the presence of more than 120 other important compounds and phenol. After detection, phenol was separated by solvent extraction method, where Ethyl ether $\left(\mathrm{C}_{4} \mathrm{H}_{10} \mathrm{O}\right)$, Caustic soda $(\mathrm{NaOH})$ and Hydrochloric acid $(\mathrm{HCl})$ were used to separate phenol from BAW and then Nuclear magnetic resonance spectroscopy (NMR) was done to confirm the recovery of phenol.
\end{abstract}

Keywords: NMR; GC/MS; Biomass pyrolysis; Bio-oil production; Phenol extraction

Abbreviations: BAW: Bio-oil obtained from agricultural wastes; BHTP: Bio-oil obtained at high temperature $\left(90^{\circ} \mathrm{C}\right)$ after pyrolysis; BLTP: Bio-oil obtained at low temperature $\left(10^{\circ} \mathrm{C}\right)$ after pyrolysis.

\section{Introduction}

Bio-oil is a complex mixture which contains a large number of organic compounds, including alcohol, organic acids, phenol, aldehyde, ketone, etc. Some of these chemicals, such as phenols are important industrial raw materials and additives [1-3]. The total amount of phenolic compounds in the pyrolysis oil varies from $20.0 \%$ to $30 \%$ depending on the biomass used and operating conditions [4,5]. Biooil contains several hundreds of chemicals as a result, it exhibits some inferior properties, such as high water content, high oxygen content, high viscosity low flash point, and strong corrosiveness [6]. These drawbacks make it difficult to be directly used as a vehicle fuel. Therefore, several upgrading technologies have been developed to improve the quality of bio-oil, including catalytic hydrodeoxygenation [7-9], catalytic cracking, steam reforming, catalytic esterification, supercritical upgrading and so on [10]. Compared with phenols derived from petroleum fuel, these phenolic compounds are renewable and easily obtained. These phenols are not only used as a replacement for phenol in phenol-formaldehyde resins but also as raw materials for developing bio-based antioxidants and many other purposes [11-15]. Pyrolysis offers the cheapest route to renewable liquid fuels. Nonetheless, many aspects of the pyrolysis pathway are still under investigation. The diverse array of research into biomass pyrolysis is multi-disciplinary and multi-dimensional and includes Pyrolysis Oil (PO) characterization, kinetic studies, new distillation systems like vacuum distillations system, computational fluid dynamics, design of new reactors, new catalytic systems, microwave-assisted pyrolysis, optimizing the pyrolysis yield, process intensification, techno-economic analysis, molecular distillation system, environmental assessment, in addition to enterprise-wide and supply chain optimization [16-22]. Now-a-day biomass has an un ignorable importance in our life and in industries as an interesting renewable resource used to provide second generation of biofuels or chemicals [23-27]. Large amount and $\mathrm{CO}_{2}$ neutrality with low sulfur and nitrogen contents make biomass a sustainable and eco-friendly energy source [28-32]. Recently bio-oil has been paid attention to provide fuels and chemicals and the residues of pyrolysis could be used as soil fertilizer [33-38]. Biomass is a $\mathrm{CO}_{2} \mathrm{H}_{2}$ and syngas neutral energy source that has considerable stockpile. It can replace fossil feedstock in the production of heat, electricity, transportation fuels, chemicals, soil fertilizers and other important materials [39-41]. Liquid bio-fuels, which are considered to be substitutes for traditional fossil fuels, can be produced from biomass in different ways, such as high-pressure liquefaction, fast pyrolysis and Hydro-thermal pyrolysis [42-45]. Pyrolysis is a technology that can efficiently convert biomass stockpile into liquid biofuels. The liquid obtained from fast pyrolysis, which is also called crude bio-oil, may be used as burning oil in boilers or even as a transportation fuel after upgrading [46,47]. In fast pyrolysis, lignocellulosic molecules of biomass are rapidly decomposed to short chain molecules in the absence of oxygen [48]. Under conditions of high heating rate, short residence time, and moderate pyrolysis temperature, pyrolysis vapor and some char are generated. After condensation of the pyrolysis vapor, liquid product can be collected in a yield of up to 65$75 \%$ on a dry weight basis $[49,50]$.

\section{Experimental}

\section{Materials discarded sawdust and other reagents}

The BAW was obtained by pyrolysis of a mixture (3:1 in mass) of sawdust (Pterocarpus, Eucalyptus and Kapok ect) and $\mathrm{CaO}$. The sawdust was mixed with the water after their granulometric reduction Calcium oxide was added to this mixture. After preparation, the mixture was dried at environmental temperature for 24 hours.

\section{Production of BAW}

The BAW was produced from the pyrolysis of sawdust in the presence of $25 \% \mathrm{CaO}$ as catalyst. The biomass sample was kept inside a stainless steel reactor of pyrolysis system which was connected to two other glass chambers as shown in Figure 1. The temperature of the chamber which had biomass was increased from $30^{\circ} \mathrm{C}$ to $900^{\circ} \mathrm{C}$ with the help of electric heater, temperature controller cabinet, and

*Corresponding author: Zeban Shah, Federal University of Rio, Grande do Sul Porto Alegre, RS, Brazil, Tel: 555182634325; E-mail: zs_zaib77@yahoo.com

Received November 02, 2016; Accepted December 12, 2016; Published January 02, 2017

Citation: Shah Z, Renato CV, Marco AC, Rosangela DS (2017) Separation of Phenol from Bio-oil Produced from Pyrolysis of Agricultural Wastes. Mod Chem Appl 5: 199. doi: 10.4172/2329-6798.1000199

Copyright: @ 2017 Shah Z, et al. This is an open-access article distributed under the terms of the Creative Commons Attribution License, which permits unrestricted use, distribution, and reproduction in any medium, provided the original author and source are credited. 
two condensers. In this system through biomass was converted to biogas and then condensed to bio-oil at temperatures $90^{\circ} \mathrm{C}$ and $10^{\circ} \mathrm{C}$ respectively. The two condensed fractions BHTP and BLTP were collected and introduced for further analysis (Figure 2).

\section{GC-MS analysis of BHTP and BLTP (BAW)}

The bio-oil identification were performed on a GC Agilent series
6890 with an Agilent mass selective detector of series 5973, a capillary polar wax column, polyethylene glycol (PEG)-coated (length of $30 \mathrm{~m}$, internal diameter of $0.25 \mathrm{~mm}$, and film thickness of $0.25 \mu \mathrm{m}$ ) Chromatographic conditions: Injection volume of $0.2 \mu \mathrm{L}$, oven at $40^{\circ} \mathrm{C}$ ( $1 \mathrm{~min}$ ) $6^{\circ} \mathrm{C} \mathrm{min}^{-1}$ up to $300^{\circ} \mathrm{C}$ (10/Min) split mode with a ratio of $100: 1$ and injection temperature of $290^{\circ} \mathrm{C}$. Time taken was 54.3 minutes, $\mathrm{He}$ (helium) as carrier gas with a flow rate of $2.9 \mathrm{~mL} \mathrm{~min}^{-1}$ (Figure 3).

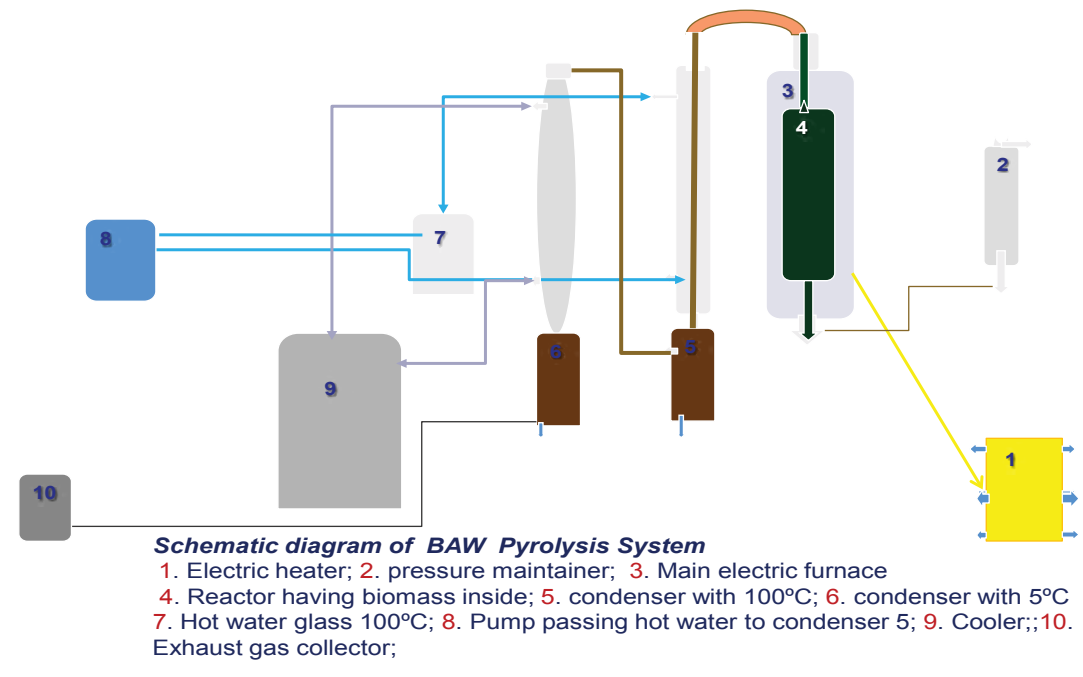

Figure 1: Schematic diagram of Biomass pyrolysis system.

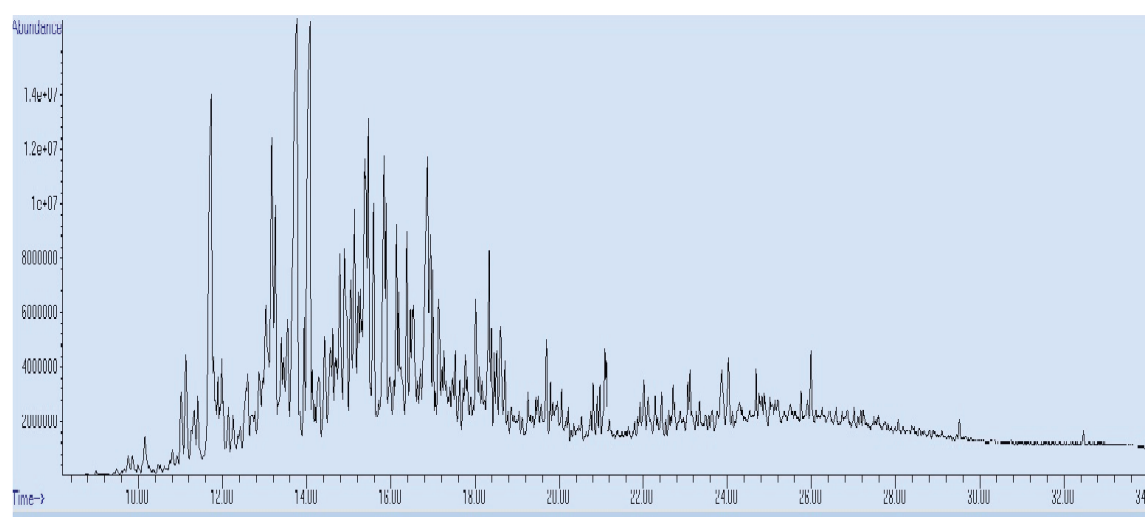

Figure 2: Chromatograph operated in splitless mode.

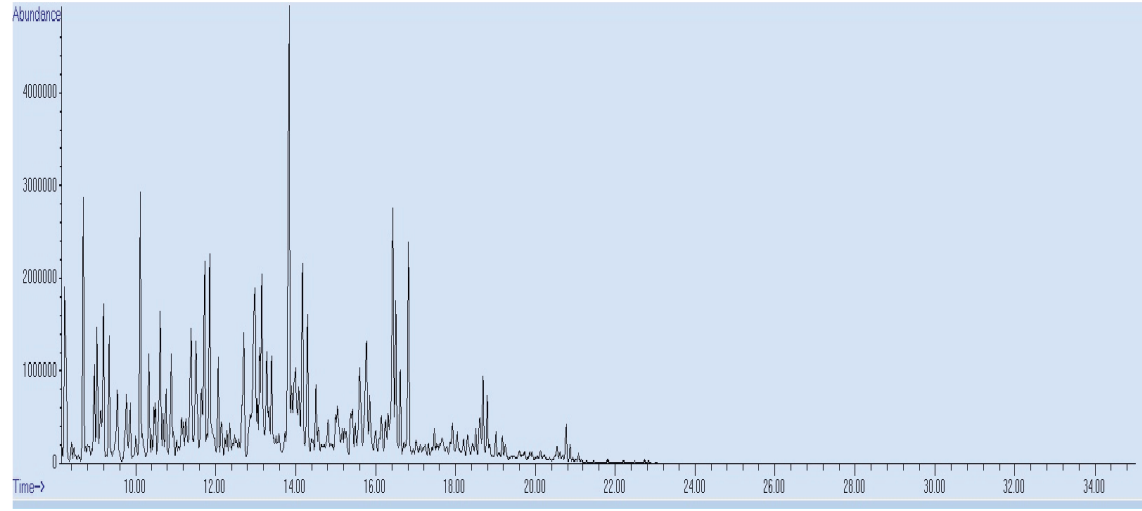

Figure 3: Chromatograph operated in split mode. 


\section{Extraction of phenols}

A feasible separation route to isolate phenolic fraction from biooil was investigated. A certain amount of Ethyl ether $\left(\mathrm{C}_{4} \mathrm{H}_{10} \mathrm{O}\right)$ and $20 \mathrm{ml}$ of $10 \%$ solution of Caustic soda $(\mathrm{NaOH})$ was added to bio-oil to separate the water and phenol from the bio-oil by reacting with phenol to form Sodium phenoxide $\left(\mathrm{NaOH}+\mathrm{C}_{6} \mathrm{H}_{6} \mathrm{O}=\mathrm{NaOC}_{6} \mathrm{H}_{5}+\mathrm{H}_{2} \mathrm{O}\right)$. The phase splitting was initiated and two phases occurred, a bottom aqueous layer which was a little bit clear and transparent brown compared to an upper layer that was very viscous and dark. The formed bottom layer was separated and $20 \mathrm{ml}$ of $10 \%$ solution of $\mathrm{HCl}$ was added to it, to react with $\mathrm{NaOC}_{6} \mathrm{H}_{5}$ in solution to form $\mathrm{NaCl}$ and phenol $\left(\mathrm{NaOC}_{6} \mathrm{H}_{5}+\mathrm{HCl}=\mathrm{C}_{6} \mathrm{H}_{6} \mathrm{O}+\mathrm{NaCl}\right)$ and after $\mathrm{pH}$ test, the water was evaporated through hot air and the remaining compound (phenol) was washed with liquid $\mathrm{NaCl}$ and introduced to $\mathrm{NMR}$ to confirm the recovery of phenol from bio-oil as shown in Figure 4 and a simple sketch in Figure 5. While reactions and mechanisms are shown in Figure 6.

\section{Results and Discussion}

\section{Chemical composition of BAW}

BAW was a dark and sticky liquid mixture of more than 120 of organic compounds. The compounds detected in BAW can be classified into hydrocarbons, alcohols, phenol, ethers, aldehydes, ketones, carboxylic acids, and other esters. But large peaks of GC/ MS mostly showed aromatic, aliphatic, and cyclic hydrocarbons while small peaks showed other groups, Library match was used for identification of compounds based on probability score and each compound was detected very clearly and with a high probability value. According to GC/MS analysis summarized in Tables 1 and 2, mostly aromatics and aliphatic groups were enriched in the sample. After GC/MS analysis, each peak of chromatogram was matched with library one by one, where different peaks showed different Aliphatic and Aromatic compounds as well as other important compounds like phenol and ketone (Figures 7 and 8).

\section{Hydrocarbons in BAW}

Tables 2 and 3 show $\mathrm{C}_{8}-\mathrm{C}_{17}$ hydrocarbons and other classes were enriched in the BAW. As shown in Table 1. Aliphatic and aromatic hydrocarbons with $\mathrm{C}_{8}-\mathrm{C}_{17}$ are predominant in the BAW sample with a \% area of 53.99

Alcohols, Aldehydes, Ketones, Ethers, Esters, Phenols, and Nitrogenous compound also present in BAW while in these classes, Phenols and ketones occupied more space compared to others as shown in Table 4 and 5. Above Figure 9 is the graphical representation of both fractions BHTP (blue) and BLTP (red) Show \% area of all groups present in BAW.

\section{Catalytic optimization}

Numerous reaction were carried out in which the amount of

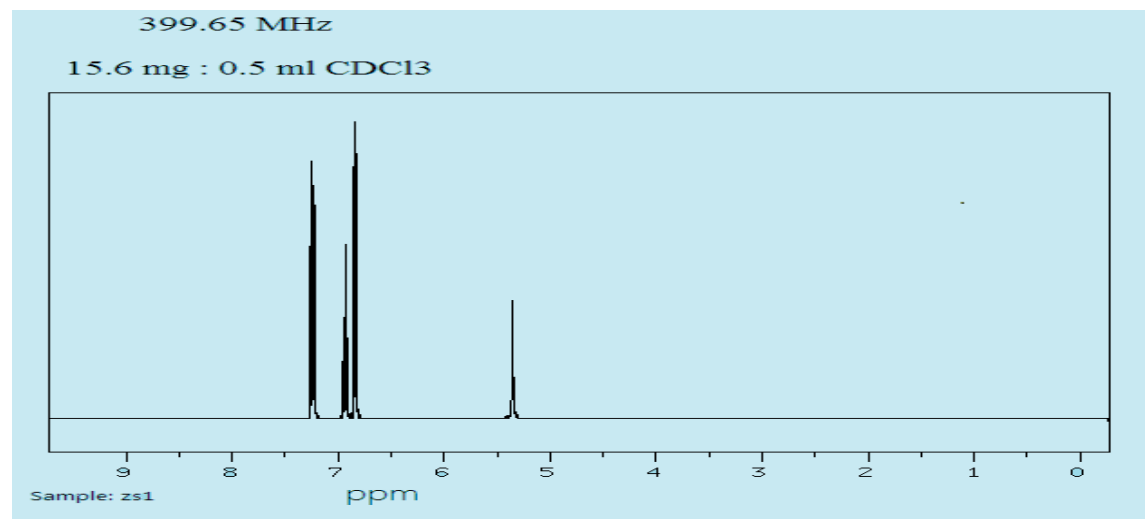

Figure 4: NMR chromatogram showing the presence of phenol.

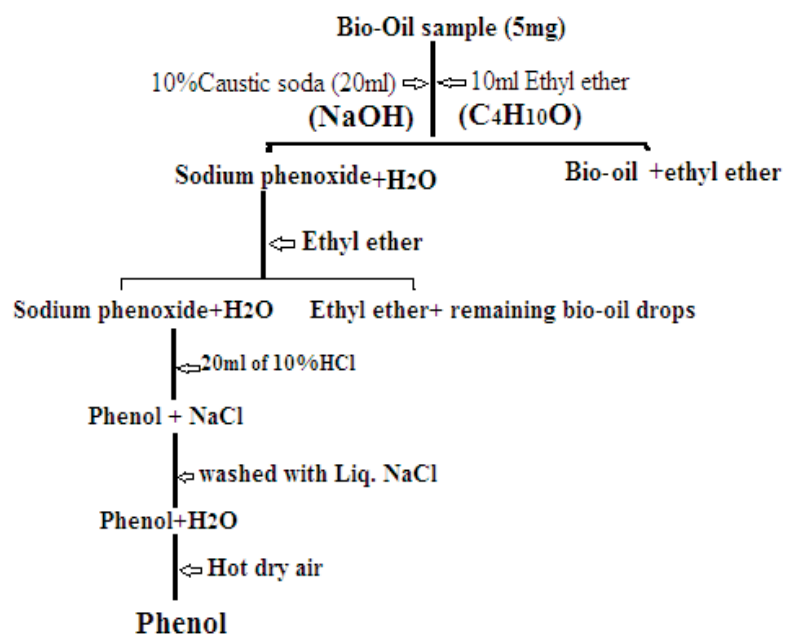

Figure 5: The simple sketch above shows how to recover phenol from bio-oil step by step. 

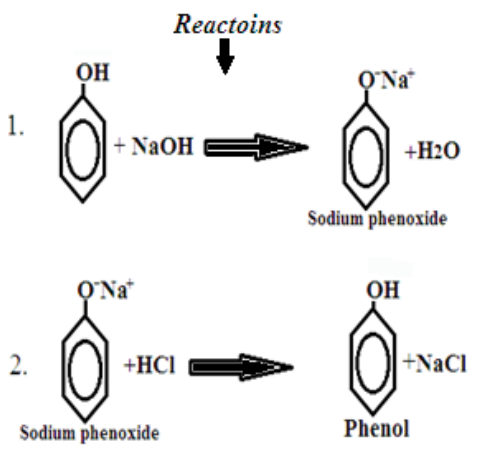

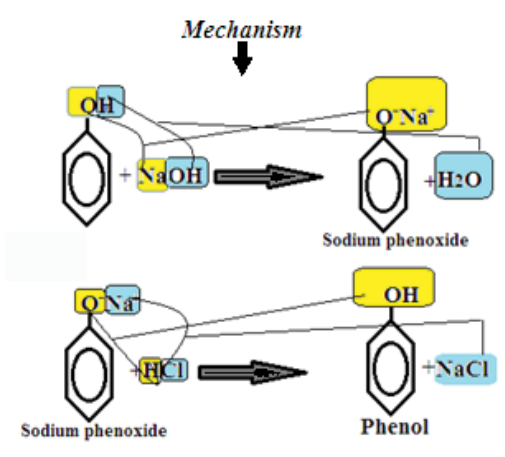

Figure 6: The reaction steps and mechanism above show how $\mathrm{NaOH}$ reacts with Phenol.

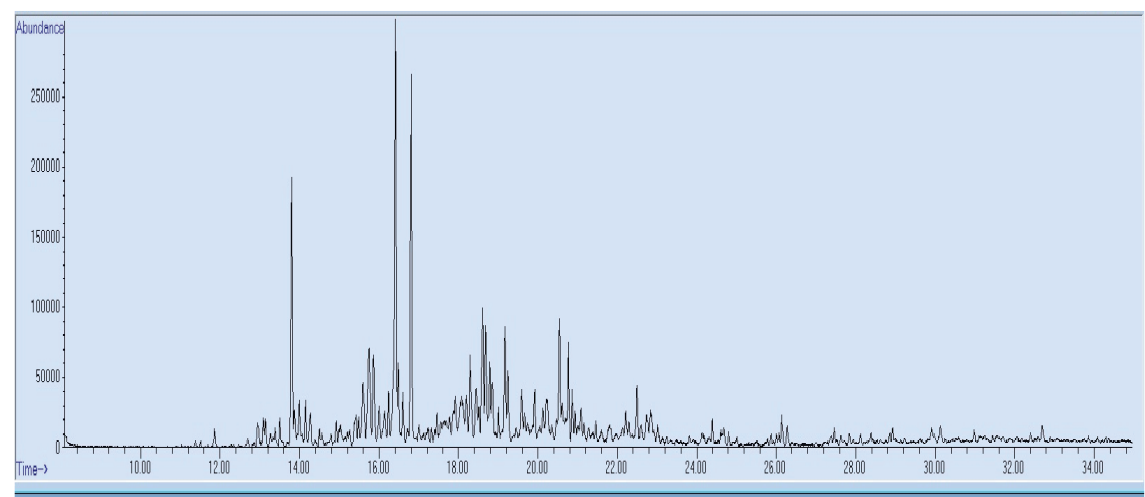

Figure 7: Shows only aliphatic hydrocarbon peaks.

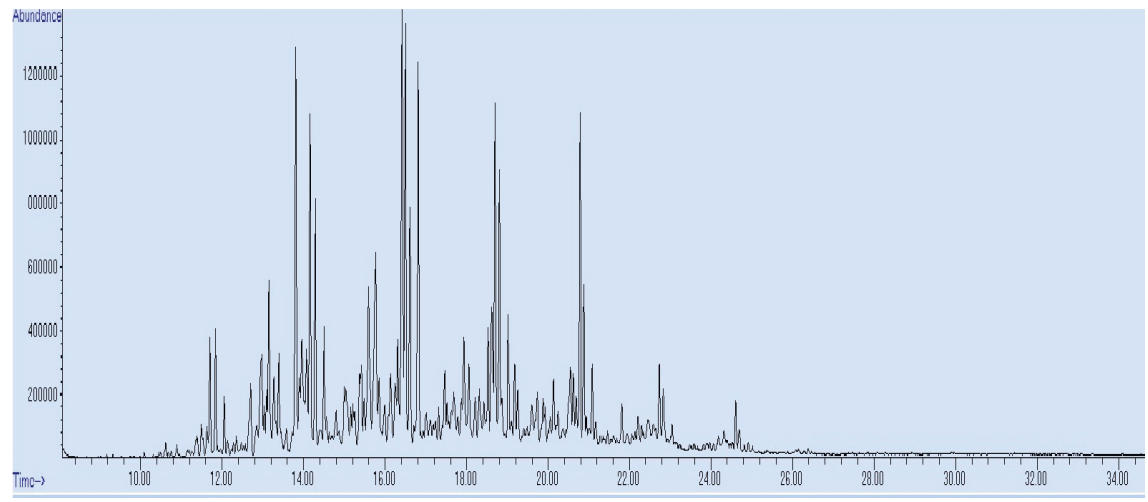

Figure 8: Shows only aromatic hydrocarbon peaks.

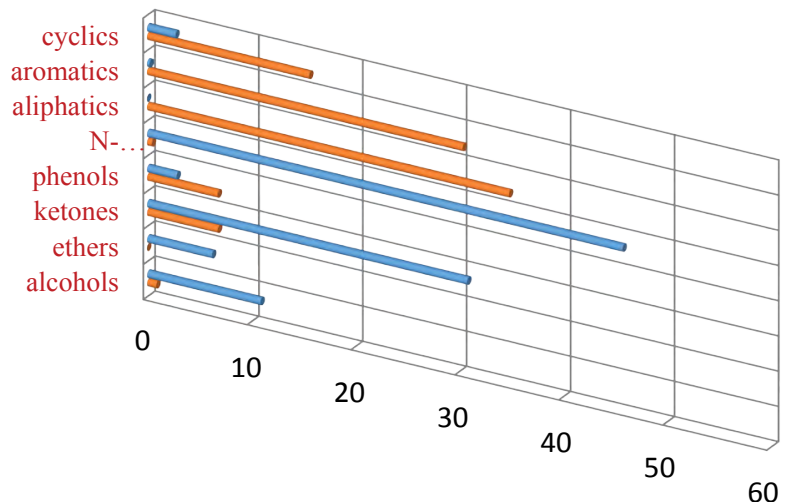

Figure 9: \% area of compounds in BHTP (blue) and BLTP (red) 
Citation: Shah Z, Renato CV, Marco AC, Rosangela DS (2017) Separation of Phenol from Bio-oil Produced from Pyrolysis of Agricultural Wastes. Mod Chem Appl 5: 199. doi: 10.4172/2329-6798.1000199

Page 5 of 8

\begin{tabular}{|c|c|c|c|}
\hline NO & Compound's Name & Formula & Retention time \\
\hline 1 & Benzene, 1-ethyl-3-methyl & $\mathrm{C}_{9} \mathrm{H}_{12}$ & 8.18 \\
\hline 2 & Benzene, 1,2,3-trimethyl & $\mathrm{C}_{9} \mathrm{H}_{12}$ & 9.00 \\
\hline 3 & Decane & $\mathrm{C}_{10} \mathrm{H}_{22}$ & 9.15 \\
\hline 4 & 2-Decene, (Z) & $\mathrm{C}_{10} \mathrm{H}_{20}$ & 9.32 \\
\hline 5 & cis-3-Decene & $\mathrm{C}_{10} \mathrm{H}_{20}$ & 9.52 \\
\hline 6 & Benzene, 1,2,3-trimethyl & $\mathrm{C}_{9} \mathrm{H}_{12}$ & 9.72 \\
\hline 7 & Benzene, 2-propenyl & $\mathrm{C}_{9} \mathrm{H}_{10}$ & 9.82 \\
\hline 8 & Indane & $\mathrm{C}_{9} \mathrm{H}_{10}$ & 10.17 \\
\hline 9 & Indene & $\mathrm{C}_{9} \mathrm{H}_{8}$ & 10.30 \\
\hline 10 & Benzene, butyl & $\mathrm{C}_{10} \mathrm{H}_{14}$ & 10.52 \\
\hline 11 & Benzene, 1,2-diethyl & $\mathrm{C}_{10} \mathrm{H}_{14}$ & 10.72 \\
\hline 12 & Benzene, 1-methyl-4-(1-methylethyl) & $\mathrm{C}_{10} \mathrm{H}_{14}$ & 11.30 \\
\hline 13 & Undecane & $\mathrm{C}_{11} \mathrm{H}_{24}$ & 11.65 \\
\hline 14 & 5-Undecene, $(Z)$ & $\mathrm{C}_{11} \mathrm{H}_{22}$ & 11.80 \\
\hline 15 & Benzene, 4-ethenyl-1,2-dimethyl & $\mathrm{C}_{10} \mathrm{H}_{12}$ & 12.65 \\
\hline 16 & 1H-Indene, 2,3-dihydro-5-methyl & $\mathrm{C}_{10} \mathrm{H}_{12}$ & 12.90 \\
\hline 17 & 7-Methyl-1,2,3,5,8,8a-hexahydronaphthalene & $\mathrm{C}_{11} \mathrm{H}_{16}$ & 13.00 \\
\hline 18 & 2-Methylindene & $\mathrm{C}_{10} \mathrm{H}_{10}$ & 13.10 \\
\hline 19 & Benzene, pentyl & $\mathrm{C}_{11} \mathrm{H}_{16}$ & 13.13 \\
\hline 20 & Naphthalene, 1,2,3,4-tetrahydro & $\mathrm{C}_{10} \mathrm{H}_{12}$ & 13.25 \\
\hline 21 & Benzene, (1-methylbutyl) & $\mathrm{C}_{11} \mathrm{H}_{16}$ & 13.38 \\
\hline 22 & Naphthalene & $\mathrm{C}_{10} \mathrm{H}_{8}$ & 13.75 \\
\hline 23 & 3-Dodecene, (Z) & $\mathrm{C}_{12} \mathrm{H}_{24}$ & 13.90 \\
\hline 24 & 3-Dodecene, (Z) & $\mathrm{C}_{12} \mathrm{H}_{24}$ & 14.05 \\
\hline 25 & Dodecane & $\mathrm{C}_{12} \mathrm{H}_{26}$ & 14.15 \\
\hline 26 & 3-Dodecene, (E) & $\mathrm{C}_{12} \mathrm{H}_{24}$ & 14.25 \\
\hline 27 & 3-Dodecene, (Z) & $\mathrm{C}_{12} \mathrm{H}_{24}$ & 14.50 \\
\hline 28 & 2-Ethyl-2,3-dihydro-1H-indene & $\mathrm{C}_{11} \mathrm{H}_{14}$ & 14.95 \\
\hline 29 & Benzene, hexyl & $\mathrm{C}_{12} \mathrm{H}_{18}$ & 15.55 \\
\hline 30 & Benzene, (1-methylpentyl) & $\mathrm{C}_{12} \mathrm{H}_{18}$ & 15.70 \\
\hline 31 & 2-Tridecene, $(Z)$ & $\mathrm{C}_{13} \mathrm{H}_{26}$ & 16.30 \\
\hline 32 & Naphthalene, 1-meth yl & $\mathrm{C}_{11} \mathrm{H}_{10}$ & 16.35 \\
\hline 33 & Tridecane & $\mathrm{C}_{13} \mathrm{H}_{28}$ & 16.45 \\
\hline 34 & 3-Tridecene, (E) & $\mathrm{C}_{13} \mathrm{H}_{26}$ & 16.55 \\
\hline 35 & 1H-Indene, 1-ethylidene & $\mathrm{C}_{11} \mathrm{H}_{10}$ & 16.75 \\
\hline 36 & Benzene, heptyl & $\mathrm{C}_{13} \mathrm{H}_{20}$ & 17.90 \\
\hline 37 & 1-Methyl-2-n-hexylbenzene & $\mathrm{C}_{13} \mathrm{H}_{20}$ & 18.00 \\
\hline 38 & Naphthalene, 2-ethyl & $\mathrm{C}_{12} \mathrm{H}_{12}$ & 18.60 \\
\hline 39 & 3-Tetradecene, (E) & $\mathrm{C}_{14} \mathrm{H}_{28}$ & 18.50 \\
\hline 40 & Tetradecane & $\mathrm{C}_{14} \mathrm{H}_{30}$ & 18.65 \\
\hline 41 & 3-Tetradecene, (E) & $\mathrm{C}_{14} \mathrm{H}_{28}$ & 19.00 \\
\hline 42 & Naphthalene, 1,7-dimethyl & $\mathrm{C}_{12} \mathrm{H}_{12}$ & 19.15 \\
\hline 43 & Pentadecane & $\mathrm{C}_{15} \mathrm{H}_{32}$ & 20.72 \\
\hline 44 & n-Nonylcyclohexane & $\mathrm{C}_{15} \mathrm{H}_{30}$ & 21.80 \\
\hline 45 & Hexadecane & $\mathrm{C}_{16} \mathrm{H}_{34}$ & 22.70 \\
\hline 46 & Cyclopentane, undecyl & $\mathrm{C}_{16} \mathrm{H}_{32}$ & 22.80 \\
\hline 47 & Heptadecane & $\mathrm{C}_{17} \mathrm{H}_{36}$ & 24.58 \\
\hline
\end{tabular}

Table 1: Aliphatic and Aromatic hydrocarbons identified in BAW.

\begin{tabular}{|c|c|c|c|}
\hline NO & Compound's Name & Formula & Retention time (Min) \\
\hline 1 & pentanol & $\mathrm{C}_{5} \mathrm{H}_{12} \mathrm{O}$ & 9.50 \\
\hline 2 & pentanone & $\mathrm{C}_{5} \mathrm{H}_{10} \mathrm{O}$ & 7.63 \\
\hline 3 & hexanone & $\mathrm{C}_{6} \mathrm{H}_{12} \mathrm{O}$ & 9.50 \\
\hline 4 & octanone & $\mathrm{C}_{8} \mathrm{H}_{16} \mathrm{O}$ & 20.83 \\
\hline 5 & heptanone & $\mathrm{C}_{7} \mathrm{H}_{14} \mathrm{O}$ & 15.77 \\
\hline 6 & Hexenol & $\mathrm{C}_{6} \mathrm{H}_{12} \mathrm{O}$ & 13.89 \\
\hline 7 & cyclopentanone & $\mathrm{C}_{5} \mathrm{H}_{8} \mathrm{O}$ & 11.23 \\
\hline 8 & cyclopentanone, $\mathrm{C}_{1}$ & $\mathrm{C}_{6} \mathrm{H}_{10} \mathrm{O}$ & 13.63 \\
\hline
\end{tabular}

\begin{tabular}{|c|c|c|c|}
\hline 9 & cyclopentanone, $\mathrm{C}_{2}$ & $\mathrm{C}_{7} \mathrm{H}_{12} \mathrm{O}$ & 16.03 \\
\hline 10 & cyclohexenone, $\mathrm{C}_{1}$ & $\mathrm{C}_{7} \mathrm{H}_{10} \mathrm{O}$ & 19.77 \\
\hline 11 & heptanone & $\mathrm{C}_{7} \mathrm{H}_{14} \mathrm{O}$ & 15.77 \\
\hline 12 & cyclopentenone, $\mathrm{C}_{3}$ & $\mathrm{C}_{8} \mathrm{H}_{12} \mathrm{O}$ & 22.57 \\
\hline 13 & acetophenone & $\mathrm{C}_{8} \mathrm{H}_{8} \mathrm{O}$ & 24.97 \\
\hline 14 & cyclohexenyl, ethanone & $\mathrm{C}_{8} \mathrm{H}_{12} \mathrm{O}$ & 26.03 \\
\hline 15 & cyclopentenone, $\mathrm{C}_{4}$ & $\mathrm{C}_{9} \mathrm{H}_{14} \mathrm{O}$ & 26.83 \\
\hline 16 & cyclohexanone, ethylidene & $\mathrm{C}_{8} \mathrm{H}_{12} \mathrm{O}$ & 28.17 \\
\hline 17 & cyclopentenone, $\mathrm{C}_{3}$ methylene & $\mathrm{C}_{9} \mathrm{H}_{12} \mathrm{O}$ & 31.50 \\
\hline 18 & indenone, hexahydro & $\mathrm{C}_{9} \mathrm{H}_{12} \mathrm{O}$ & 37.50 \\
\hline 19 & ethane, diethoxy & $\mathrm{C}_{6} \mathrm{H}_{14} \mathrm{O}_{2}$ & 9.23 \\
\hline 20 & furanmethanol & $\mathrm{C}_{5} \mathrm{H}_{6} \mathrm{O}_{2}$ & 17.37 \\
\hline 21 & furan, $\mathrm{C}_{2}$ & $\mathrm{C}_{6} \mathrm{H}_{8} \mathrm{O}$ & 22.97 \\
\hline 22 & phenol & $\mathrm{C}_{6} \mathrm{H}_{6} \mathrm{O}$ & 23.37 \\
\hline 23 & phenol, $\mathrm{C}_{1}$ & $\mathrm{C}_{7} \mathrm{H}_{8} \mathrm{O}$ & 24.97 \\
\hline 24 & pyrrole & $\mathrm{C}_{4} \mathrm{H}_{5} \mathrm{~N}$ & 9.63 \\
\hline 25 & pyrrole, $\mathrm{C}_{1}$ & $\mathrm{C}_{5} \mathrm{H}_{7} \mathrm{~N}$ & 10.43 \\
\hline 26 & piperidine, $\mathrm{C}_{1}$ & $\mathrm{C}_{6} \mathrm{H}_{13} \mathrm{~N}$ & 11.37 \\
\hline 27 & piperidine, $\mathrm{C}_{1}$ & $\mathrm{C}_{6} \mathrm{H}_{13} \mathrm{~N}$ & 11.50 \\
\hline 28 & piperidine, $\mathrm{C}_{2}$ & $\mathrm{C}_{7} \mathrm{H}_{15} \mathrm{~N}$ & 15.37 \\
\hline 29 & pyridine, $\mathrm{C}_{1}$ & $\mathrm{C}_{6} \mathrm{H}_{7} \mathrm{~N}$ & 15.50 \\
\hline 30 & pyridine, $\mathrm{C}_{3}$ & $\mathrm{C}_{8} \mathrm{H}_{11} \mathrm{~N}$ & 21.10 \\
\hline 31 & imidazole, $\mathrm{C}_{4}$ & $\mathrm{C}_{7} \mathrm{H}_{12} \mathrm{~N}_{2}$ & 15.77 \\
\hline 32 & pyrazine, $\mathrm{C}_{2}$ & $\mathrm{C}_{6} \mathrm{H}_{8} \mathrm{~N}_{2}$ & 16.43 \\
\hline 33 & pyrazine, $\mathrm{C}_{3}$ & $\mathrm{C}_{7} \mathrm{H}_{10} \mathrm{~N}_{2}$ & 21.23 \\
\hline 34 & pyrazine, $\mathrm{C}_{4}$ & $\mathrm{C}_{8} \mathrm{H}_{12} \mathrm{~N}_{2}$ & 25.37 \\
\hline 35 & pyrazine, $\mathrm{C}_{5}$ & $\mathrm{C}_{9} \mathrm{H}_{14} \mathrm{~N}_{2}$ & 29.50 \\
\hline 36 & pyrazine, $\mathrm{C}_{6}$ & $\mathrm{C}_{11} \mathrm{H}_{18} \mathrm{~N}_{2}$ & 29.90 \\
\hline 37 & pyrrolidinone, $\mathrm{C}_{2}$ & $\mathrm{C}_{6} \mathrm{H}_{11} \mathrm{NO}$ & 26.83 \\
\hline 38 & piperidinone, $\mathrm{C}_{4}$ & $\mathrm{C}_{9} \mathrm{H}_{17} \mathrm{NO}$ & 27.23 \\
\hline 39 & pentanamide, $\mathrm{C}_{1}$ & $\mathrm{C}_{6} \mathrm{H}_{13} \mathrm{NO}$ & 28.30 \\
\hline 40 & pyrazine, $\mathrm{C}_{6}$ & $\mathrm{C}_{10} \mathrm{H}_{16} \mathrm{~N}_{2}$ & 33.37 \\
\hline 41 & imidazole, $\mathrm{C}_{3}$ & $\mathrm{C}_{6} \mathrm{H}_{10} \mathrm{~N}_{2}$ & 31.23 \\
\hline 42 & pyrazole, $\mathrm{C}_{3}$ & $\mathrm{C}_{6} \mathrm{H}_{10} \mathrm{~N}_{2}$ & 33.23 \\
\hline 43 & pyrazole, $\mathrm{C}_{4}$ & $\mathrm{C}_{7} \mathrm{H}_{12} \mathrm{~N}_{2}$ & 33.37 \\
\hline 44 & imidazole, $\mathrm{C}_{4}$ & $\mathrm{C}_{7} \mathrm{H}_{12} \mathrm{~N}_{2}$ & 33.50 \\
\hline 45 & pyridine, $\mathrm{C}_{1}$ propenyl & $\mathrm{C}_{10} \mathrm{H}_{13} \mathrm{~N}$ & 34.97 \\
\hline 46 & pyrrolidinone, $\mathrm{C}_{2}$ methylidene & $\mathrm{C}_{7} \mathrm{H}_{11} \mathrm{NO}$ & 36.18 \\
\hline
\end{tabular}

Table 2: Compounds of other groups detected in BAW.

\begin{tabular}{|c|c|c|c|}
\hline NO & Name & Formula & Retention time (Min) \\
\hline 1 & Undecane & $\mathrm{C}_{11} \mathrm{H}_{24}$ & 11.65 \\
\hline 2 & 5-Undecene, $(\mathrm{Z})$ & $\mathrm{C}_{11} \mathrm{H}_{22}$ & 11.80 \\
\hline 3 & 3-Dodecene, $(\mathrm{Z})$ & $\mathrm{C}_{12} \mathrm{H}_{24}$ & 13.90 \\
\hline 4 & 3-Dodecene, $(\mathrm{Z})$ & $\mathrm{C}_{12} \mathrm{H}_{24}$ & 14.05 \\
\hline 5 & Dodecane & $\mathrm{C}_{12} \mathrm{H}_{26}$ & 14.15 \\
\hline 6 & 3-Dodecene, $(\mathrm{E})$ & $\mathrm{C}_{12} \mathrm{H}_{24}$ & 14.25 \\
\hline 7 & 3-Dodecene, $(\mathrm{Z})$ & $\mathrm{C}_{12} \mathrm{H}_{24}$ & 14.50 \\
\hline 8 & 2-Tridecene, $(\mathrm{Z})$ & $\mathrm{C}_{13} \mathrm{H}_{26}$ & 16.30 \\
\hline 9 & Tridecane & $\mathrm{C}_{13} \mathrm{H}_{28}$ & 16.45 \\
\hline 10 & 3-Tridecene, $(\mathrm{E})$ & $\mathrm{C}_{13} \mathrm{H}_{26}$ & 16.55 \\
\hline 11 & 3-Tetradecene, $(\mathrm{E})$ & $\mathrm{C}_{14} \mathrm{H}_{28}$ & 18.50 \\
\hline 13 & Tetradecane & $\mathrm{C}_{14} \mathrm{H}_{30}$ & 18.65 \\
\hline 14 & 3-Tetradecene, $(\mathrm{E})$ & $\mathrm{C}_{14} \mathrm{H}_{28}$ & 19.00 \\
\hline 15 & Pentadecane & $\mathrm{C}_{15} \mathrm{H}_{32}$ & 20.72 \\
\hline 16 & Hexadecane & $\mathrm{C}_{16} \mathrm{H}_{34}$ & 22.70 \\
\hline & Tahle & \\
\hline
\end{tabular}

Table 3: Shows Aliphatic compounds identified in BAW. 


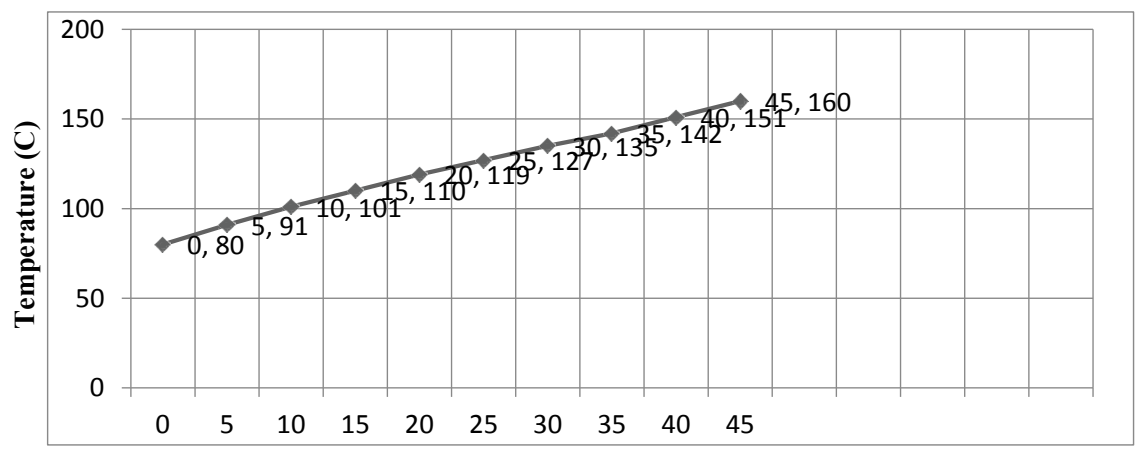

Volume (ml)

Figure 10: Distillation curve for BLTP

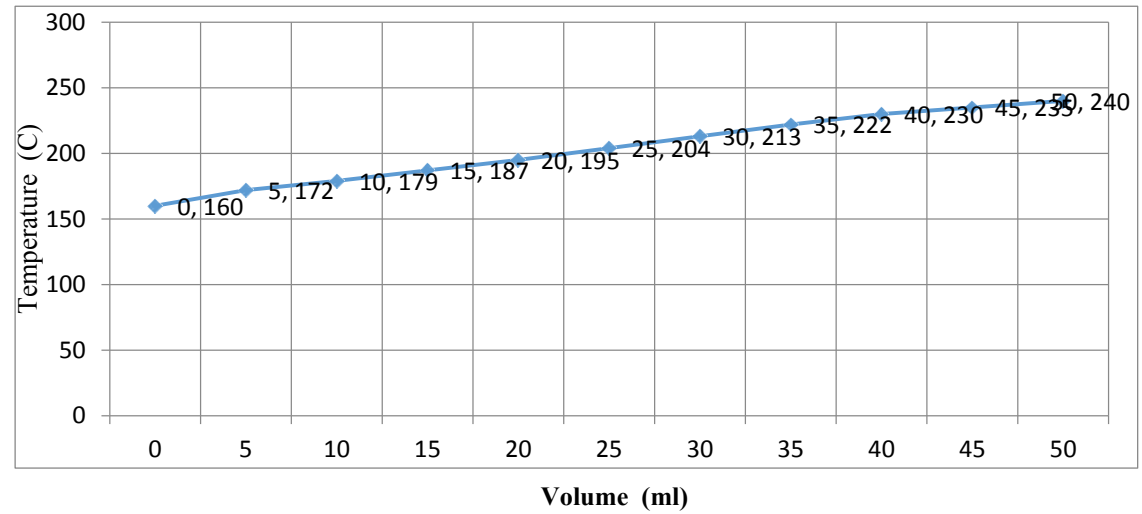

Figure 11: Distillation curve for BHTP.

\begin{tabular}{|c|c|c|c|}
\hline NO & Name & Formula & Retention time (min) \\
\hline 1 & Benzene, 4-ethenyl-1,2-dimethyl & $\mathrm{C}_{10} \mathrm{H}_{12}$ & 12.65 \\
\hline 2 & 1H-Indene, 2,3-dihydro-5-methyl & $\mathrm{C}_{10} \mathrm{H}_{12}$ & 12.90 \\
\hline 3 & $\begin{array}{c}\text { 7-Methyl-1,2,3,5,8,8a- } \\
\text { hexahydronaphthalene }\end{array}$ & $\mathrm{C}_{11} \mathrm{H}_{16}$ & 13.00 \\
\hline 5 & 2-Methylindene & $\mathrm{C}_{10} \mathrm{H}_{10}$ & 13.10 \\
\hline 6 & Benzene, pentyl & $\mathrm{C}_{11} \mathrm{H}_{16}$ & 13.13 \\
\hline 7 & Naphthalene, 1,2,3,4-tetrahydro & $\mathrm{C}_{10} \mathrm{H}_{12}$ & 13.25 \\
\hline 8 & Benzene, (1-methylbutyl) & $\mathrm{C}_{11} \mathrm{H}_{16}$ & 13.38 \\
\hline 9 & Naphthalene & $\mathrm{C}_{10} \mathrm{H}_{8}$ & 13.75 \\
\hline 10 & 2-Ethyl-2,3-dihydro-1H-indene & $\mathrm{C}_{11} \mathrm{H}_{14}$ & 14.95 \\
\hline 11 & Benzene, hexyl & $\mathrm{C}_{12} \mathrm{H}_{18}$ & 15.55 \\
\hline 12 & Benzene, (1-methylpentyl) & $\mathrm{C}_{12} \mathrm{H}_{18}$ & 15.70 \\
\hline 13 & Naphthalene, 1-methyl & $\mathrm{C}_{11} \mathrm{H}_{10}$ & 16.35 \\
\hline 14 & 1H-Indene, 1-ethylidene & $\mathrm{C}_{11} \mathrm{H}_{10}$ & 16.75 \\
\hline 15 & Benzene, heptyl & $\mathrm{C}_{13} \mathrm{H}_{20}$ & 17.90 \\
\hline 16 & 1-Methyl-2-n-hexylbenzene & $\mathrm{C}_{13} \mathrm{H}_{20}$ & 18.00 \\
\hline 17 & Naphthalene, 2-ethyl & $\mathrm{C}_{12} \mathrm{H}_{12}$ & 18.60 \\
\hline 18 & Naphthalene, 1,7-dimethyl & $\mathrm{C}_{12} \mathrm{H}_{12}$ & 19.15 \\
\hline 19 & n-Nonylcyclohexane & $\mathrm{C}_{15} \mathrm{H}_{30}$ & 21.80 \\
\hline
\end{tabular}

Table 4: Shows Aromatic compounds identified in BAW.

biomass was remained the same while catalyst $(\mathrm{CaO})$ was varying to choose a suitable ratio of catalyst with biomass at which maximum pyrolysis yield while less amount of residue is obtained. At different ratio of $\mathrm{CaO}$ and biomass the pyrolysis reactions were carried out, finally 3:1 of biomass and $\mathrm{CaO}$ respectively were found proper for a good pyrolysis yield (Figures 10-12).

\begin{tabular}{|c|c|c|}
\hline \multirow{2}{*}{ Different Classes of compounds } & \multicolumn{2}{|c|}{$\%$ Area } \\
\cline { 2 - 3 } & BLTP & BHTP \\
\hline Alcohols & 0.889 & 11.110 \\
\hline Aldehydes & 0.501 & 1.123 \\
\hline Ketones & 7.02 & 25.827 \\
\hline Ethers & n.d. & 6.263 \\
\hline Esters & n.d. & 1.923. \\
\hline Phenols & 7.109 & 4.858 \\
\hline Nitrogenous & 0.354 & 40.779 \\
\hline Aromatics hydrocarbons & 33.001 & 2.246 \\
\hline Cyclic hydrocarbons & 17.420 & 3.096 \\
\hline Aliphatic hydrocarbons & 35.121 & 0.101 \\
\hline
\end{tabular}

Table 5: \% area of deferent classes of compounds identified in BAW.

\section{Conclusions}

After different process and analyzing techniques, more than 120 compounds were detected in the BAW. Among them, aromatic, aliphatic and cyclic hydrocarbons, especially alkanes, alkenes and benzene containing compounds were dominant but other important compounds like phenol were also present. A laboratory scale effort is made in this work to recover phenol from BAW, however, to improve efficiency, this process can be successfully applied in largescale operations because phenol is an important compound used in the preparation of resins, dyes, explosives, lubricants, pesticides and plastics. It is indirectly useful in the preparation of plywood. Phenol 


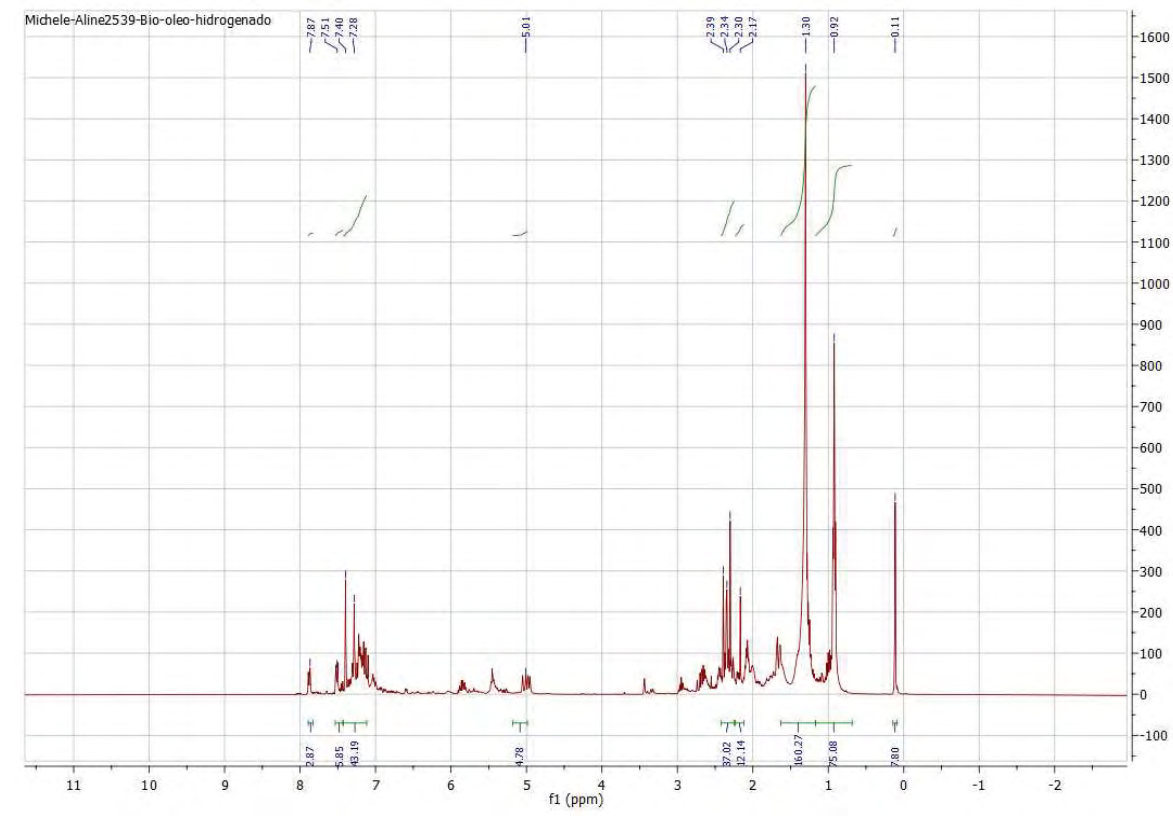

Figure 12: BAW H.NMR spectra.

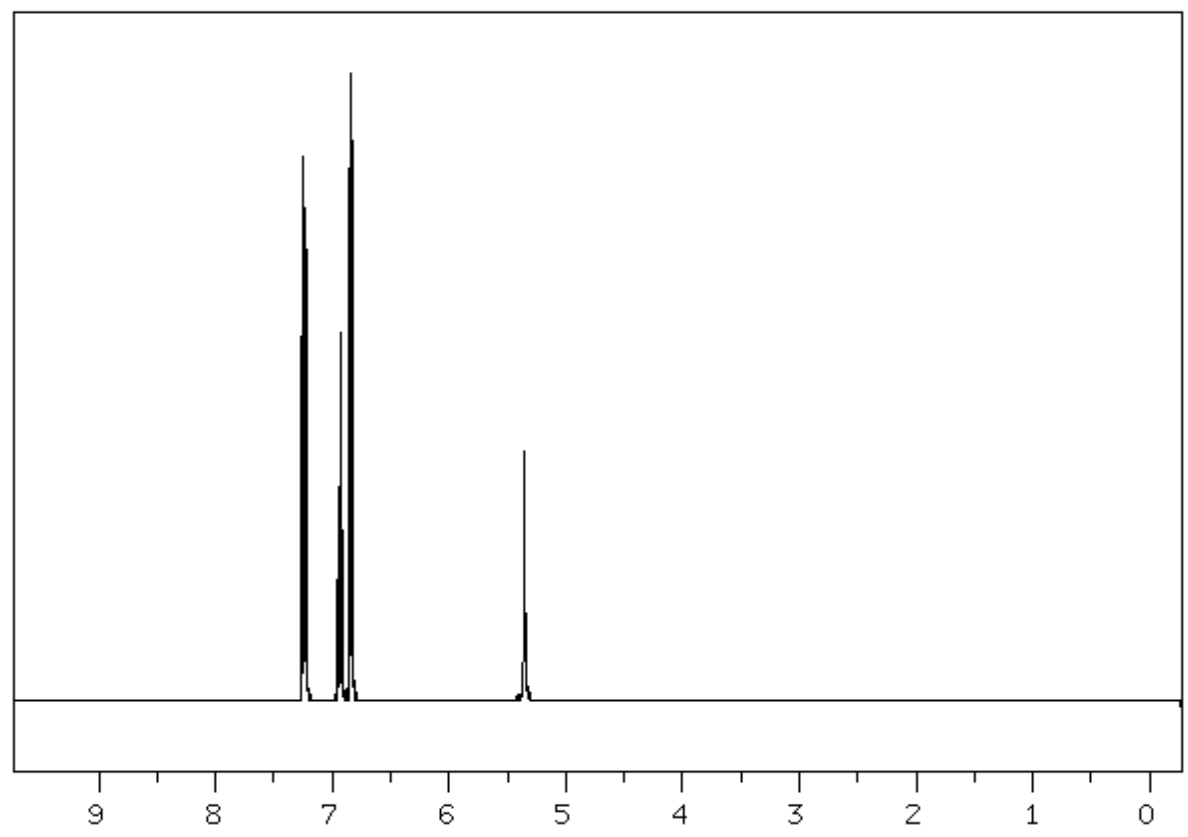

Figure 13: Phenols H.NMR spectra.

is also used as an organic solvent to dissolve other alcohols as well as for medicinal purposes. Future work is going to test light fraction (80$\left.160^{\circ} \mathrm{C}\right)$ in diesel engine and heavy fraction $\left(160-240^{\circ} \mathrm{C}\right)$ in a jet engine by mixing them in normal diesel fuels and aviation fuels respectively (Figure 13).

\section{Acknowledgements}

The financial support of CNPq, Brazil was acknowledged.

\section{References}

1. Mortensen PM, Grunwaldt JD, Jensen PA, Knudsen KG, Jensen AD, et al. (2011)A review of catalytic upgrading of bio-oil to engine fuels. Appl. Catal. A 407: 1-19.
2. Bridgwater AV (2012) Review of fast pyrolysis of biomass and product upgrading. Biomass Bioenergy 38: 68-94.

3. Kwon KC, Mayfield H, Marolla T, Nichols B, Mashburn M (2011)Catalytic deoxygenation of liquid biomass for hydrocarbon fuels. Renew Energy 36: 907-915.

4. Choudhary TV, Phillips CB (2011) Renewable fuels via catalytichydrodeoxygenation. Appl. Catal. A 397: 1-12.

5. Wang S, Cai Q, Wang X, Guo Z, Luo Z, et al. (2013) Bio-gasoline production from cocracking of hydroxypropanone and ethanol. Fuel Process Technol 111 86-93.

6. Wang S, Cai Q, Guo Z, Wang Y, Wang X, et al. (2012) Renewable gasoline produced by cocracking of methanol and ketones in bio-oil. BioResources 7 : 5019-5031. 
Citation: Shah Z, Renato CV, Marco AC, Rosangela DS (2017) Separation of Phenol from Bio-oil Produced from Pyrolysis of Agricultural Wastes. Mod Chem Appl 5: 199. doi: 10.4172/2329-6798.1000199

Page 8 of 8

7. Wang S, Li X, Guo L, Luo Z (2012)Experimental research on acetic acid steamreforming over $\mathrm{Co}-\mathrm{Fe}$ catalysts and subsequent density functional theorystudies. Int J Hydrogen Energy. 37: 11122-11131.

8. Trane R, Dahl S, Rasmussen MS, Jensen AD (2012) Catalytic steamreforming of bio-oil. Int J Hydrogen Energy 37: 6447-6472.

9. Wang JJ, Chang J, Fan J (2010) Upgrading of bio-oil by catalytic esterification anddetermination of acid number for evaluating esterification degree. Energy Fuel 24: 3251-3255.

10. Miao S, Shanks BH (2009) Esterification of biomass pyrolysis model acids oversulfonic acid-functionalized mesoporous silicas. Appl Catal A 359: 113-120.

11. Li W, Pan C, Zhang Q, Liu Z (2011) Upgrading of high-boiling fraction of bio-oil in supercritical methanol and reaction network. Bioresour. Technol. 102: 4884-4889.

12. Li W, Pan C, Sheng L, Liu Z (2011) Upgrading of high boiling fraction of bio-oil in supercritical methanol. Bioresour. Technol. 102: 9223-9228.

13. Kanaujia PK, Sharma YK, Garg MO, Tripathi D (2014) Review of analyticalstrategies in the production and upgrading of bio-oils derived fromlignocellulosic biomass. J Anal Appl Pyrol 105: 55-74.

14. Lin SH, Juang RS (2009) Adsorption of phenol and its derivatives from water usingsynthetic resins and low-cost natural adsorbents A review. J Environ Manage 90: 1336-1349.

15. Hu G, Li J, Zeng G (2013) Recent development in the treatment of oily sludge frompetroleum industry A review. J Hazard Mater 261: 470-490.

16. Ma L, Wang T, Liu Q, Zhang X (2012) A review of thermal-chemical conversion of lignocellulosic biomass in China. Biotechnol Adv 30: 859-873.

17. Rover MR, Brown RC (2013) Quantification of total phenols in the bio-oil using the Folin-Ciocalteu Method. J Anal Appl Pyrol. 104: 366-371.

18. Effendi A, Gerhauser H, Bridgwater AV (2008) Production of renewable phenolicresins by thermochemical conversion of biomass A review. Renew Sustain.Energy Rev. 12: 2092-116.

19. Xu J, Jiang J, Dai W, Sun Y (2010) Rice husk bio-oil upgrading by means ofphase separation and the production of esters from the water phase, andnovolac resins from the insoluble phase, Biomass Bioenergy 34: 10591063

20. Gonza J, Cruz JM (2004) Production of antioxidants fromEucalyptus globulus wood by solvent extraction of emicellulose hydrolysates. Food Chem 84: 243-251.

21. Azeez AM, Meier D, Odermatt J (2010) Willner T Fast pyrolysis ofAfrican and European lignocellulosic biomasses using $\mathrm{Py}-\mathrm{GC} / \mathrm{MS}$ and fluidized bed reactor. Energy Fuel 24: 2078-2085.

22. Ben H, Ragauskas AJ (2011) NMR characterization of pyrolysis oilsfrom Kraft lignin. Energy Fuel 25: 2322-2332.

23. Ben H, Ragauskas AJ (2013) Comparison for the compositions offast and slow pyrolysis oils by NMR Characterization. Bioresour Technol 147: 577-584.

24. Mettler MS, Vlachos DG, Dauenhauer PJ (2012) Top tenfundamental challenges of biomass pyrolysis for biofuels. Energy Environ Sci 5: 7797-7809.

25. White JE, Catallo WJ, Legendre BL (2011) Biomass pyrolysis kinetics:a comparative critical review with relevant agriculturalresidue case studies. J Anal Appl Pyrolysis 91: 1-33.

26. Papadikis K, Gu S, Bridgwater AV, Gerhauser H (2009) Application of CFD to model fast pyrolysis of biomass. Fuel Process Technol 90: 504-512.

27. Isahak WNRW, Hisham MWM, Yarmo MA, Hin TY (2012) A reviewon bio-oi production from biomass by using pyrolysismethod. Renew Sust Energ Rev 16: $5910-5923$

28. Motasemi F, Afzal MT (2013) A review on the microwave-assisted pyrolysis technique. Renew Sust Energ Rev 28: 317-330.

29. Borges FC, Du Z, Xie Q, Trierweiler JO, Cheng Y, et al. (2014) Fast microwave assisted pyrolysis of biomass usingmicrowave absorbent. Bioresour Technol Mar 156: 267-274.

30. Akhtar J, Amin NS (2012) A review on operating parameters foroptimum liquid oil yield in biomass pyrolysis. Renew Sust Energ Rev 16: 5101-5109.

31. Sadhukhan J (2012) Multiscale simulation for high efficiency biodiesel process intensification. Proceedings of the 22nd European Symposium on Compute
Aided ProcessEngineering, London, In: Bogle IDL, Fairweather $\mathrm{M}$ (eds.) Computer aided chemical engineering 30: 1023-1027.

32. Brown TR, Thilakaratne R, Brown RC, Hu G (2013) Technoeconomicanalysis of biomass to transportation fuels andelectricity via fast pyrolysis and hydroprocessing. Fuel 106: 463-469.

33. Wright MM, Daugaard DE, Satrio JA, Brown RC (2010) Technoeconomicanalysis of biomass fast pyrolysis totransportation fuels. Fuel 89: 2-10.

34. Gebreslassie BH, Slivinsky M, Wang B, You F (2013) Life cycleoptimization for sustainable design and operations ofhydrocarbon biorefinery via fast pyrolysis, hydrotreating andhydrocracking. Comput Chem Eng 50: 71-91.

35. Kim J, Realff MJ, Lee JH, Whittaker C, Furtner L, et al. (2011) Design of biomass processing network for biofuel production using an MILP model. Biomass Bioenerg. 35: 853-871.

36. Akgul O, Shah N, Papageorgiou LG (2012) Economic optimisation of a UK advanced biofuel supply chain economic optimisation of a UK advanced biofuel supply chain. Biomass Bioenerg 41: 57-72.

37. Braimakisa K, Atsoniosa K, Panopoulos KD, Karellas S, Kakaras E (2014) Economic evaluation of decentralized pyrolysisfor the production of biooil as an energy carrier forimproved logistics towards a large centralized gasificationplant. Renew Sust Energ Rev 35: 57-72.

38. Huber GW, Iborra S, Corma A (2006) Synthesis of transportation fuels from biomass chemistry catalysts and engineering Chem Rev 106: 4044-4098.

39. Zeng FX, Liu WJ, Jiang H, Yu HQ (2011) Separation of phthalateesters from bio-oil derived from rice husk by a basification-acidificationprocess and column chromatography. Bioresour. Technol 102: 1982-1987.

40. Xu CB, Etcheverry T (2008) Hydro-liquefaction of woody biomass in sub- and supercriticalethanol with iron-based catalysts. Fuel 87: 335-345.

41. Mohan D, Pittman CU, Steele PH (2006) Pyrolysis of wood/biomass for bio-oil A critical review. Energy Fuels 20: 848-889.

42. Bridgwater AV, Meier D, Radlein D (1999) An overview of fast pyrolysis of biomass. Org Geochem 30: 1479-1493.

43. Bridgwater AV (2012) Review of fast pyrolysis of biomass and product upgrading. Biomass Bioenergy 38: 68-94.

44. Wang SR,Wang YR, Cai QJ, Wang XY (2014) Multi-step separation of monophenols and pyrolytic lignins from the water-insoluble phase of bio oil. Sep Purif Technol 122: 248-255.

45. Vitasari CR, Meindersma GW, de Haan AB (2011) Water extraction of pyrolysis oil the first step for the recovery of renewable chemicals. Bio resour Technol 107: 204-210.

46. Pollard AS, Rover MR, Brown RC (2012) Characterization of bio-oil recovered as stage fractions with unique chemical and physical properties. J Anal App Pyrolysis 93: 129-138.

47. Chen CA, Pakdel H, Roy C (1997) Separation of phenols from Eucalyptus wood tar. Biomass Bioenergy 13: 25-37.

48. Guo ZG, Wang SR, Gu YL, Xu GH (2010) Separation characteristics of biomass pyrolysis oil in molecular distillation. Sep Purif Technol. 76: 52-57.

49. Zilnik LF, Jazbinsek A (2012) Recovery of renewable phenolic fraction from pyrolysis oil. Sep Purif sTechnol. 86: 157-170.

50. Wei Y, Lei HW, Wang L, Zhu L (2014) Liquid-liquid extraction of biomass pyrolysis bio-oil. Energy Fuels 28: 1207-1212. 